
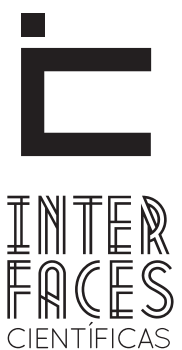

SAÚDE E AMBIENTE

ISSN IMPRESSO 2316-3313

E - ISSN 2316-3798

DOI - 10.17564/2316-3798.2018v6n2p43-52

\title{
FATORES DE RISCO PARA A HIPERTENSÃO ARTERIAL EM COMUNIDADE PESQUEIRA NORDESTINA BRASILEIRA ${ }^{1}$
}

\author{
RISK FACTORS FOR ARTERIAL HYPERTENSION IN NORTHEAST BRAZILIAN FISHERY COMMUNITY \\ FACTORES DE RIESGO PARA LA HIPERTENSIÓN ARTERIAL EN COMUNIDAD PESQUERA NORDESTINA BRASILEÑA
}

Carla Grasiela Santos de Oliveira²

Maria Regivalda Santana de Souza
Guilherme Mota da Silva ${ }^{3}$

Francisco Prado Reis ${ }^{5}$

\section{RESUMO}

Este trabalho teve por objetivo analisar os fatores de risco para a o desenvolvimento da hipertensão arterial (HA), em uma comunidade pesqueira. Foi realizado inquérito socioambiental e cultural, anamnese clínica e avaliação antropometria, em uma amostra de 370 sujeitos de uma comunidade pesqueira de Aracaju, Sergipe. Sessenta e um por cento da população estudada eram de pescadores, pertencentes à classe socioeconômica desfavorecida. A prevalência de sobrepeso e obesidade foi respectivamente $35 \%$ e $23 \%$, com maior frequência no sexo feminino e $40 \%$ dos entrevistados apresentaram algum tipo de HA. A hipertensão é uma doença existente na comunidade estudada e os fatores desencadeadores deste processo são o baixo nível socioeconômico, hábitos de vida não saudáveis, sobrepeso e obesidade. Diante deste cenário é necessário programar estratégias de educação e saúde capazes de controlar ou reduzir os fatores de risco, a hipertensão arterial e suas comorbidades.

\section{PALAVRAS-CHAVE}

Hipertensão Arterial. Pescadores. Hábitos de Vida.

1 Artigo baseado na dissertação de mestrado de Carla Grasiela Santos de Oliveira, intitulada "Avaliação das condições de saúde e qualidade de vida de comunidade pesqueira sergipana”, defendida em 2012 no Programa de Pós-Graduação em Saúde e Ambiente da Universidade Tiradentes. 


\section{ABSTRACT}

This work aimed to analyze the risk factors for the development of arterial hypertension (AH) in a fishing community. It was carried out environmental and cultural inquiry, clinical history and assessment anthropometry, in a sample of 370 subjects of a fishing community in Aracaju, Sergipe. Sixty-one percent of the population studied were fishermen, belonging to the lower socioeconomic classes. The prevalence of overweight and obesity were respectively $35 \%$ and $23 \%$ more frequently in females and $40 \%$ of respondents had some type of $\mathrm{AH}$. Hypertension is a con-

\section{RESUMEN}

Este trabajo tuvo por objetivo analizar los factores de riesgo para el desarrollo de la hipertensión arterial (HA) en una comunidad pesquera. Se realizó encuesta socioambiental y cultural, bien como examen clínico y la antropométrico, en una muestra de 370 sujetos de una comunidad de pescadores en Aracaju, Sergipe. Sesenta y uno por ciento de la población estudiada eran pescadores, pertenecientes a las clases socioeconómicas más bajas. La prevalencia de sobrepeso y obesidad fueron, respectivamente, el $35 \%$ y el $23 \%$ con más frecuencia en las mujeres y el $40 \%$ de los encuestados tenía algún tipo de HTA. La hipertensión dition found in the community under study and the factors that trigger this process are the low socioeconomic status, unhealthy living habits, overweight and obesity. Given this scenario it is necessary to program education and health strategies to control or reduce risk factors, high blood pressure and its comorbidities

\section{KEYWORDS}

Arterial Hypertension. Fishermen. Habits of Life. arterial es una patología existente en la comunidad estudiada y desencadena este proceso son el estatus socioeconómico bajo, hábitos de vida poco saludables, el sobrepeso y la obesidad. Ante este escenario, es necesario programar las estrategias de educación y de salud para controlar o reducir los factores de riesgo, hipertensión arterial y sus comorbilidades.

\section{PALABRAS CLAVE}

Hipertensión arterial. Pescadores. Hábitos de vida. 


\section{INTRODUÇÃ̃O}

A hipertensão arterial sistêmica (HAS) é uma doença crônica não transmissível, inserida no grupo de doenças cardiovasculares. É um problema de saúde pública por sua magnitude, risco e dificuldades no seu controle (SCHIMIDT et al., 2011; WHO, 2011). Além destes aspectos, trata-se de um dos mais importantes fatores para o desenvolvimento do acidente vascular cerebral (AVC), infarto do miocárdio e síndromes próprias que apresentam características peculiares (WHO, 2011; NOBRE et al., 2013). A HAS é uma condição clínica multifatorial, geralmente assintomática, associada a alterações funcionais, estruturais e metabólitas dos órgãos alvo com elevado risco para a morbimortalidade (SBC, 2010). Esta patologia tem se estabelecido de forma crescente na sociedade e a suas principais repercussões está atrelado à incapacidade e morte, quando a doença não é diagnosticada ou controlada (MENDONÇA et al., 2012).

Estima-se que a doença atinja aproximadamente um bilhão de pessoas no mundo, sendo responsável por mais de 7,1 milhões de mortes ao ano. Numericamente isto representa 57 milhões anos de vida perdidos por incapacidade (DALY). Nos indivíduos com mais de 60 anos a prevalência chega a $50 \%$ e nos adultos a sua frequência é em torno de $20 \%$.

Um inquérito realizado pela Organização Mundial de Saúde no ano de 2008 revelou que a prevalência da doença foi maior na África com $46 \%$ em relação às Américas, com um percentual de $35 \%$. Esta análise permitiu identificar que hipertensão arterial atinge com maior frequência as mulheres (WHO, 2015). No Brasil a doença atinge em média $25 \%$ da população brasileira, mais de $50 \%$ são idosos, $5 \%$ dos 70 milhões de crianças e adolescentes são acometidos pela doença e o diagnóstico ocorre com maior frequência em mulheres em todas as faixas de idade (BRASIL, 2014).

Os estudos epidemiológicos têm identificado a associação positiva entre doença e os fatores de risco modificáveis tais como sobrepeso, obesidade, estresse, dislipidemia, sedentarismo, tabagismo, anticoncepcionais, características sociodemográficas, etilismo, ingestão de uma alimentação rica em sódio e gorduras e, os não modificáveis como etnia e história familiar. 0 somatório destes fatores de risco tem contribuído para o aumento da morbimortalidade e o controle dos modificáveis por meio da adoção de um estilo de vida saudável tem impactado para melhor qualidade de vida dos indivíduos hipertensos (MACHADO; PIRES; LOBÃO, 2012; NASCENTE et al., 2010).

Apesar de consolidada a relação entre $\mathrm{HA}$ e os fatores nutricionais, ainda não são bem esclarecidos os mecanismos de atuação destes sobre a elevação da pressão arterial. Conhece-se, no entanto, os efeitos de uma dieta saudável sobre o comportamento dos níveis pressóricos (SACKS et al., 2001). Evidências cientificas confirmam que os padrões alimentares errôneos e determinados fatores dietéticos são elementos propulsores para o desencadeamento desta patologia. Por outo lado, uma alimentação adequada do ponto de vista da segurança alimentar é um a mediada protetiva na prevenção de doenças crônicas. Deste modo, em função da relação intrínseca entre a prevalência de HA e hábitos de vida não saudáveis e, tendo em vista a relevância da patologia, o objetivo desta pesquisa foi identificar os fatores de risco para a hipertensão arterial em uma comunidade pesqueira.

\section{MATERIAL E MÉTODOS}

Trata-se de uma pesquisa descritiva, de corte transversal, abordagem quantitativa e com uma população delimitada. O local em estudo foi especificamente a comunidade pesqueira inserida no Povoado Mosqueiro situado na cidade de Aracaju/SE que possui uma população de 4885 habitantes (SIAB, 2011). 
Figura 1 - Localização da Comunidade Mosqueiro no Município de Aracaju, SE

Fonte: Autores.

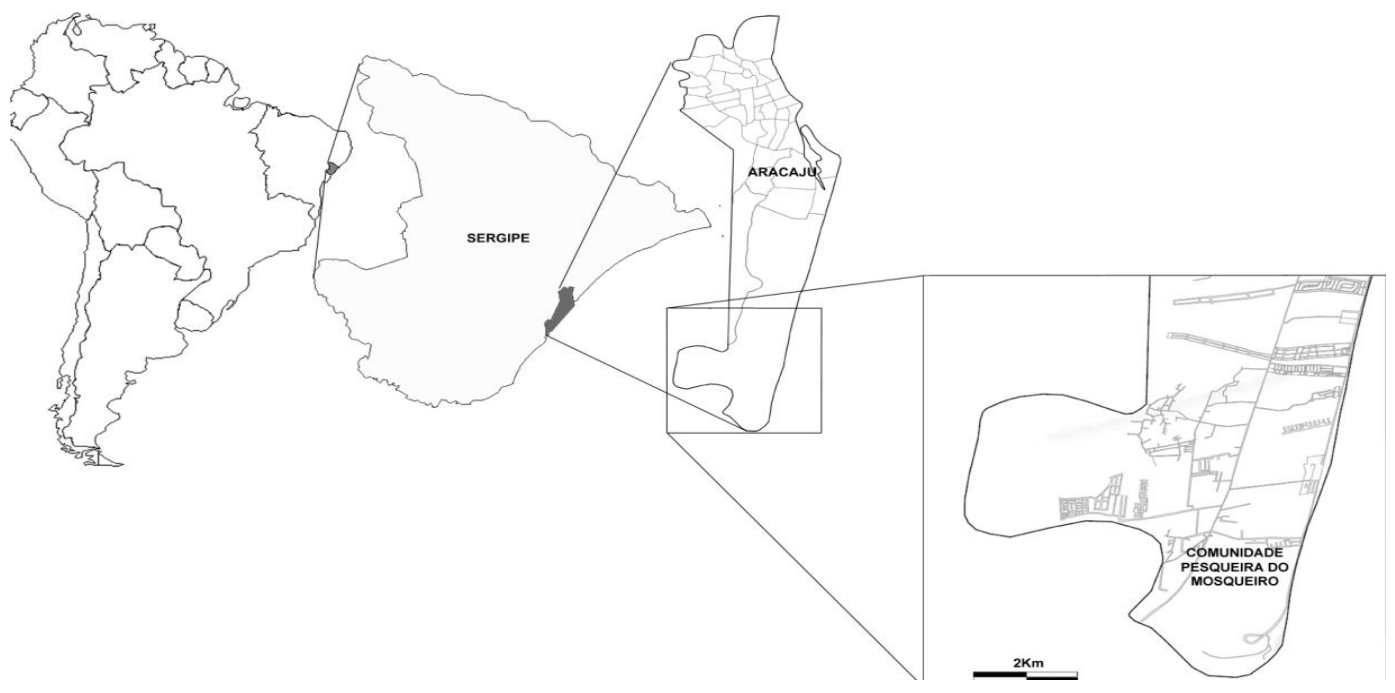

Para o cálculo amostral da população foi aplicado à metodologia de Barbetta (2001) com 5\% de significância. Os participantes da pesquisa indivíduos são pertencentes à comunidade de pescadores, com idade a partir de 18 anos, selecionados de forma aleatória de acordo com as microáreas determinadas pela Equipe da Estratégia da Saúde Família (ESF) local.

A pesquisa foi dividida em duas etapas: a aplicação de um formulário socioambiental e cultural, contendo as seguintes variáveis: renda familiar, escolaridade, condições de moradia e saneamento básico, hábitos de vida e morbidade referia e frequência de alimentos consumidos. A segunda etapa foi composta pela anamnese clínica, com a ausculta dos níveis pressóricos, frequência cardíaca, frequência respiratória e classificação do Índice de Massa Corpórea (IMC) para predizer a gordura corporal.

A pressão arterial foi aferida em repouso, utilizando o estetoscópio e esfigmomanômetro das marcas $\mathrm{BD}^{\circledast}$ e Premium ${ }^{\circledR}$ devidamente calibrados, e os valores foram anotados em milímetros de mercúrio $(\mathrm{mmHg})$. A mensuração da frequência cardíaca foi realizada durante um minuto, na artéria radial ou na carótida quando na presença de lesões. A massa corporal foi determinada em quilogramas, utilizando uma balança eletrônica digital, com capacidade máxima de $150 \mathrm{Kg}$. Para a mensuração da estatura (metros) foi utilizado um antropômetro vertical milimetrado, com extensão de $2 \mathrm{~m}$ e escala de $0,5 \mathrm{~cm}$. 0 índice de massa corporal (IMC) foi calculado, relacionando o peso $(\mathrm{Kg})$ e a altura ao quadrado (metros) e os parâmetros utilizados foram os recomendados pela Organização Mundial da Saúde (WHO, 2000).

Os dados foram coletados no período de maio a novembro de 2011, nas residências dos pesquisados. $\mathrm{Na}$ análise dos dados foi utilizada a estatística descritiva com medidas de frequência e teste de correlação Pearson, com um nível de significância de $95 \%$. Para conduzir a análise dos resultados foi utilizado o software Statistical Package for the Social Sciences versão 15. A pesquisa foi aprovada pelo Comitê de Ética em Pesquisa com Seres Humanos da Universidade Tiradentes, sob o parecer $n^{\circ} 291110 \mathrm{R}$ e obedeceu à Resolução n¹96/96 e suas complementares do Con- 
selho Nacional de Saúde/Ministério da Saúde que dispõem sobre Diretrizes e Normas regulamentares na Pesquisa com Seres Humanos. A autorização foi obtida por meio da assinatura pelos sujeitos, do Termo de Consentimento Livre e Esclarecido.

\section{RESULTADOS}

Participaram da pesquisa 370 indivíduos, sendo $61,1 \%$ mulheres e $38,9 \%$ homens, com idade variável entre 18 e 89 anos (42,03 anos $\mathbf{1 1 6 , 6 5}$ ). Deste total, $61,6 \%$ desenvolvem atividades com a pesca, $30,3 \%$ outras atividades tais como do lar, pedreiro, vigilante, técnico de enfermagem, professor e 8,1\% dos entrevistados são ex-pescadores. Em relação ao gênero a pesca foi mais predominante no sexo masculino. Aqueles que laboram na atividade pesqueira, $50 \%$ referem pescar peixe, coletar marisco e caranguejo, 39,1\% peixe e mariscos e 10,9\% unicamente peixe. No que se refere à renda familiar, $65 \%$ percebem entre 1 a 2 salários mínimos, 22,7\% possui uma renda mensal menor que 1 salário mínimo e 12, 1\% percebem mais de 4 salários mínimos. Os dados de escolaridade demonstram que $52,2 \%$ possuem ensino fundamental incompleto, $15,9 \%$ médio completo incompleto, 12,7\% médio incompletos, 7,3\% fundamental completo, $1,9 \%$ superior completo e $1,4 \%$ superior incompleto.

No que diz respeito à doença referida, 9,2\% dos participantes referiram apresentar doenças cardiovasculares, artrose, diabetes, osteoporose, alergia respiratória e reumatismo. Na avaliação auscultatória da pressão arterial, 97,8\% participaram do procedimento. Do total de sujeitos avaliados, foi constatado que $33,9 \%$ estavam com a P.A dentro dos parâmetros de normalidade (P.A Sistólica/P.A Diastólica 120X $80 \mathrm{~mm} \mathrm{Hg}$ ), 14, 6\% num patamar ótimo, menor que a referência, mas não hipotenso, 40,1\%, apresentaram algum grau de hipertensão e 10, 2\% no limítrofe, tendenciado para um quadro de hipertensão. Ao relacionar os estágios da hipertensão arterial e média de idade, verificou-se que a elevação dos níveis pressóricos é diretamente proporcional ao avanço da idade. A análise do gênero revelou que as mulheres foram as mais acometidas pela doença (Tabela 1).

Tabela 1 - Distribuição da classificação da HAS segundo o gênero e idade nos indivíduos da Comunidade Pesqueira. Aracaju-SE, 2012

\begin{tabular}{|c|c|c|c|c|c|c|}
\hline & \multirow{2}{*}{$\begin{array}{l}\text { Ótima } \\
\text { n (\%) }\end{array}$} & \multirow{2}{*}{$\begin{array}{l}\text { Normal } \\
\mathrm{n}(\%)\end{array}$} & \multirow{2}{*}{$\begin{array}{l}\text { Limítrofe } \\
\mathrm{n}(\%)\end{array}$} & \multicolumn{3}{|c|}{ Hipertensão } \\
\hline & & & & $\begin{array}{l}\text { Estágio I } \\
\mathrm{n}(\%)\end{array}$ & $\begin{array}{l}\text { Estágio II } \\
\mathrm{n}(\%)\end{array}$ & $\begin{array}{l}\text { Estágio III } \\
\mathrm{n}(\%)\end{array}$ \\
\hline Homens & $8(2,2)$ & $49(13,6)$ & $13(3,6)$ & $29(7,7)$ & $23(6,3)$ & $16(4,4)$ \\
\hline Mulheres & $45(12,4)$ & $76(20,3)$ & $24(6,8)$ & $43(11,9)$ & $22(6,1)$ & $14(3,9)$ \\
\hline Total (362)* & $53(14,6)$ & $125(33,9)$ & $37(10,4)$ & $72(19,6)$ & $45(12,4)$ & $30(8,3)$ \\
\hline Média de Idade ${ }^{\star \star}$ & $\begin{array}{l}33,0^{\mathrm{a}} \\
( \pm 13,8)\end{array}$ & $\begin{array}{l}36,2^{\mathrm{a}} \\
( \pm 14,4)\end{array}$ & $\begin{array}{l}45,6^{b} \\
( \pm 15,9)\end{array}$ & $\begin{array}{l}44,6^{b} \\
( \pm 16,1)\end{array}$ & $\begin{array}{l}52,0^{c} \\
( \pm 16,2)\end{array}$ & $\begin{array}{l}54,7^{c} \\
( \pm 15,5)\end{array}$ \\
\hline
\end{tabular}

**Médias com a mesma letra não são estatisticamente diferentes

Fonte: Dados da pesquisa 
$\mathrm{Na}$ avaliação da frequência respiratória aproximadamente $100 \%$ apresentaram movimento respiratório dentro dos padrões de normalidades e quando questionados sobre a presença de cefaleia $71,9 \%$ referiram a presença deste sintoma.

Na mensuração do peso e altura para compor o IMC, aproximadamente $97 \%$ dos pesquisados participaram da avaliação antropométrica. Os dados revelaram que $37,3 \%$ dos pesquisados são eutróficos, $35 \%$ estão com sobrepeso e $23 \%$ apresentam variados graus de obesidade. Segundo a variável gênero, o sobrepeso e a obesidade foram mais frequentes em mulheres, com o percentual $21,7 \%$ e $16,3 \%$ respectivamente. 0 sexo e o IMC apresentaram uma correlação fraca e não significativa $(r=-0,078 ; p=0,141)$, não havendo, portanto, diferenças no índice de massa corporal entre as mulheres e os homens na comunidade estudada.

Ao investigar a correlação entre frequência cardíaca e o IMC, os resultados indicaram que os indivíduos com classificados em obesidade de grau III apresentaram frequência média cardíaca mais elevada e estatisticamente diferentes dos indivíduos com outras classificações de IMC ( $p=0,0375)$ (Tabela 2)

Tabela 2 - Distribuição dos níveis de classificação de IMC nos membros da Comunidade Pesqueira, segundo o gênero e as médias das frequências cardíacas. Aracaju-SE, 2012

\begin{tabular}{|c|c|c|c|c|c|c|}
\hline \multirow[b]{2}{*}{ CLASSIFICAÇÃO IMC } & \multirow{2}{*}{$\begin{array}{l}\text { Magreza } \\
\text { grau I } \\
\text { n \% }\end{array}$} & \multirow{2}{*}{$\begin{array}{l}\text { Eutrofia } \\
n \%\end{array}$} & \multirow{2}{*}{$\begin{array}{l}\text { Sobrepeso } \\
\text { n \% }\end{array}$} & \multicolumn{3}{|c|}{ Obesidade } \\
\hline & & & & $\begin{array}{l}\text { Grau I } \\
\mathrm{n} \%\end{array}$ & $\begin{array}{l}\text { Grau II } \\
\mathrm{n} \%\end{array}$ & $\begin{array}{l}\text { Grau III } \\
\mathrm{n} \%\end{array}$ \\
\hline Mulheres & $10(2,8)$ & $74(20,6)$ & $78(21,7)$ & $44(12,2)$ & $13(3,6)$ & $2(0,5)$ \\
\hline Homens & $6(1,7)$ & $60(16,7)$ & $48(13,4)$ & $15(4,2)$ & $4(1,1)$ & $5(1,4)$ \\
\hline Total & $16(4,5)$ & $134(37,3)$ & $126(35,1)$ & $59(16,4)$ & $17(4,7)$ & $7(1,9)$ \\
\hline Freq. Cardíaca* & $68,44^{a}$ & $72,16^{\mathrm{a}}$ & $70,62^{\mathrm{a}}$ & $75,00^{\mathrm{a}}$ & $74,77^{\mathrm{a}}$ & $86,29^{b}$ \\
\hline
\end{tabular}

*Médias com a mesma letra não são estatisticamente diferentes

Fonte: Dados da pesquisa

No que se refere o estilo de vida saudáveis, $38,1 \%$ dos entrevistados declararam uso de bebida alcoólica e 17, 3\% são tabagistas. Em relação aos hábitos alimentares e a composição de sua refeição, todos os entrevistados autoreferiram que seu prato é composto em aproximadamente $80 \%$ de leguminosas e cereais, especificamente pela dupla arroz-feijão, $10 \% \mathrm{em}$ proteínas de origem animal por meio do consumo de carnes branca e vermelha e $10 \%$ em frutas, especialmente a manga e a banana disponíveis em plantações na comunidade.

\section{DISCUSSÃO}

Na presente pesquisa houve uma predominância de mulheres no estudo, isto se deve ao fato da comunidade trabalhada, ser um povoado pesqueiro, no qual os homens geralmente no período diurno desenvolvem atividades no rio ou na confecção redes de pesca próxima as embarcações e/ou reparos no barco. Este perfil de gênero é semelhante um estudo realizado em uma comunidade pesqueira do Ceará, no qual o sexo feminino foi predominante no momento da pes- 
quisa (CARVALHO; BEZERRA; PINHEIRO, 2010). A pesca exige muito esforço e oferece perigos, sendo limitante para as mulheres, justificando a predominância do gênero masculino neste tipo de atividade (SANTOS et al., 2011).

O panorama nacional revelou que $64 \%$ dos pescadores no país são do gênero masculino e a região Nordeste, é considerada a mais igualitária entre os gêneros. Contudo, especificamente ao analisar por Unidades Federativas, o Estado de Sergipe apresenta uma característica peculiar à região Nordeste, a maioria das pessoas vinculadas diretamente à pesca são mulheres, perfazendo um total de 54,9\%, do universo total, divulgando assim a importância do trabalho feminino neste setor (BRASIL, 2010). O elevado número de mulheres na pesca pode estar vinculado à atividade pesqueira dessas regiões, onde predomina a pesca artesanal e atividade de mariscagem, exercida pelas mulheres (ALENCAR; MAIA, 2011).

$\mathrm{Na}$ análise do perfil socioeconômico a maior parte dos pescadores estudados está inserida na classe social E, com um rendimento familiar mensal de aproximadamente $\mathrm{R} \$$ 1.576,00. A classificação utilizada foi baseada nos critérios do Instituto Brasileiro de Geografia e Estatística (IBGE, 2009) para definição de classes sociais. Esta estratificação econômica permite afirmar que as famílias inseridas na classe $E$ são menos favorecidas economicamente, implicando na dependência de programas assistenciais governamentais. Um estudo realizado por Paulo-Junior e outros autores (2012), revelou que 40\% dos pescadores são assistidos por programas sociais do governo e, segundo os autores, tais medidas sendo assistencialistas, conduzem a uma cultura consumista que não faz parte da realidade do pescador.

0 ensino fundamental incompleto é condição comum aos pescadores e um padrão nacional do grau de escolaridade encontrado em diversas pesquisas com esta população. A educação básica é uma précondição para educação formal e profissional, elementos necessários que contribuem para que um pescador de pequena escala de trabalho tenha instrumentos culturais, sociais e econômicos para melho- rar a sua condição de vida (ISAAC-NAHUM, 2006). A soma de pescadores com ensino fundamental incompleto e analfabeto é responsável pela baixa eficiência de políticas públicas direcionadas ao setor pesqueiro, contribuindo para a manutenção do ciclo da pobreza (ALENCAR; MAIA, 2011).

$O$ resultado da presente pesquisa demostrou que a prevalência de hipertensão arterial nesta comunidade foi elevada com um percentual de 40,1\%. Estudos realizados por pesquisadores (MARTINS et al., 2010; OLIVEIRA et al., 2013) sobre hipertensão em comunidades ribeirinhas encontraram percentuais menores $25 \%$ e $23 \%$ respectivamente. A prevalência de HAS nesta comunidade indica que as pessoas estão mais propensas a desenvolver doenças cerebrovasculares, infarto agudo do miocárdio e doenças renais, condições que elevam a morbimortalidade pela doença. A HAS é o fator de risco mais evidenciado para a mortalidade e representa uma das causas mais importantes de anos de vida com incapacidade a nível mundial (LIMA-COSTA et al., 2009).

Um fator importante observado nessa comunidade foi à presença do sobrepeso e a obesidade, importantes fatores de risco modificáveis no surgimento da HAS. Estudos sobre a tríade hipertensão, sobrepeso e obesidade, revelam que o ganho de peso ao longo da vida é um preditor para a instalação da HAS e a obesidade está associada aos níveis mais elevados da pressão do sangue nos vasos (GALVÃO; KOHLMANNJUNIOR, 2002; MOREIRA et al., 2013; SÁ et al., 2014). A eliminação destes fatores de risco, tais como tabagismo, alcoolismo, obesidade e sobrepeso podem acarretar na diminuição da frequência das doenças cardiovasculares e as estimativas apontam que para o ano 2025 a prevalência da HAS será de $29 \%$ na população mundial (TALAEI et al., 2014).

A alimentação adequada em quantidade e qualidade é um fator protetivo para o surgimento da hipertensão arterial. Desta forma, cada grupo de alimentos tem uma função específica na prevenção da HAS e na promoção da saúde, por outro lado determinados componentes dos alimentos têm uma ação desencadeadora para esta patologia. 0 uso de alimentos ricos 
em fibras foi capaz de provocar a redução dos níveis sanguíneos de colesterol total e HDL, com resultados significativos na diminuição da pressão arterial quando comparado com o grupo controle (COLIN et al., 2000). A ingestão exagerada de alimentos industrializados eleva o consumo de sódio e este microelemento é responsável pelo aumento dos níveis pressóricos (BROWN et al., 2009).

\section{CONCLUSÃO}

Os resultados obtidos nesta pesquisa revelaram que a hipertensão arterial nesta comunidade já é uma condição clínica existente em aproximadamente metade da população analisada. Um dos fatores responsáveis pelo seu surgimento é a baixa renda e o nível de escolaridade, variáveis que limitam o conhecimento sobre a doença e o acesso ao serviço de saúde e, os outros elementos desencadeadores que estão intrinsicamente ligados são alimentação inadequada, sobrepeso e a obesidade. Em função deste cenário, é necessário programar estratégias de educação e saúde, capazes de promover hábitos de vida saudáveis, além do acompanhamento com o profissional de saúde a fim de diminuir ou eliminar o surgimento da hipertensão e naqueles que já tem o diagnóstico definido as suas comorbidades associadas.

\section{REFERÊNCIAS}

ALENCAR, C.A.G.; MAIA, L.P. Perfil socioeconômico dos pescadores brasileiros. Arq. Ciên. Mar. v. 44, n. 3, p. 12-19, 2011.

\section{BARBETTA, P.A. Estatística aplicada às Ciências}

Sociais $4^{\mathrm{a}}$ edição. Florianópolis: Editora UFSC; 2001.

BRASIL. Ministério da Pesca e Aquicultura (MPA).

Boletim Estatístico da Pesca e Aquicultura. Brasília: MPA, 2010.
BRASIL. Ministério da Saúde. Portal Brasil. Hipertensão. Programa da Saúde não tem preço. 2014. Disponível em: http://www.brasil.gov.br/saude/2011/04/saude-anuncia-dados-da-hipertensaono-pais. Acesso 16 de Dezembro, 2015.

BROWN, I.J. et al. Salt intakes around the world: implications for public health. Int. J. Epidemiol. v. 38, n. 3, p. 791-813, 2009.

CARVALHO, R.M.; BEZERRA, L.N.; PINHEIRO, J.C.V. Aspectos socioeconômicos da pesca na comunidade da prainha do Canto verde - Beberibe-CE. Soc.

desenvol. rural. v. 4, n.3, p. 1-8, 2010.

COLIN, P.R. et al. The effect of dietary patterns on blood pressure control in hypertensive patients: results from the Dietary approaches to stop hypertension (DASH)

Trial. Am. J. Hypertens. v. 13, n. 9, p. 945 -955, 2000.

GALVÃO, R.; KOHLMANN - JÚNIOR, O. Hipertensão arterial no paciente obeso. Rev. Bras. Hipertens. v. 9, n. 3, p. 262-267, 2002.

IBGE, Instituto Brasileiro de Geografia e Estatística. Consulta ao endereço eletrônico http://www.ibge. gov.br acesso realizado em janeiro de 2009.

ISAAC-NAHUM, V.J. Explotação e manejo dos recursos pesqueiros do litoral amazônico: um desafio para o futuro. Cienc. Cult. v. 58, n. 3, p. 33-36, 2006.

LIMA - COSTA, M.F.F. et al. Comportamentos de saúde entre idosos hipertensos, Brasil. Rev. Saúde Pública. v. 43, supl. 2, p. 18-26, 2009.

MACHADO, M.C.; PIRES, C.G.S.; LOBÃO, W.M. Concepções dos hipertensos sobre os fatores de risco para a doença.

Ciênc. saúde coletiva. v. 17, n. 5, p. 1365-1374, 2012.

MARTINS, M.S.A. et al. Hipertensão arterial e estilo de vida em Sinop, Município da Amazônia Legal. Arq. Bras. Cardiol. v. 94, n. 5, p. 1-6, 2010. 
MENDONÇA, L.B.A.; LIMA, F.E.T.; OLIVEIRA, S.K.P. Acidente vascular encefálico como complicação da hipertensão arterial: quais são os fatores intervenientes? Esc. Anna Nery. v. 16, n. 2, p. 340-346, 2012.

MOREIRA, N.F. et al. Obesidade: principal fator de risco para hipertensão arterial sistêmica em adolescentes brasileiros participantes de um estudo de coorte. Arq. Bras. Endocrinol. Metab. v. 57, n. 7, p. 520-526, 2013.

NASCENTE, F.M.N. et al. Arterial Hypertension and its Correlation with Some Risk Factors in a Small Brazilian Town. Arq. Bras. Cardiol. v. 95, n. 4, p. 502 505, 2010.

NOBRE, F. et al. Hipertensão arterial sistêmica primária. Medicina (Ribeirão Preto). v. 46, n. 3, p. 256-272, 2013.

OLIVEIRA, B.F.A. et al. Prevalência de hipertensão arterial em comunidades ribeirinhas do Rio Madeira, Amazônia Ocidental Brasileira. Cad. Saúde Pública. v. 29, n. 8, p. 1617 - 1630, 2013.

PAULO-JÚNIOR, E.P. et al. Gestão da pesca artesanal na costa do Paraíba, Brasil: uma abordagem utilizando o processo analítico hierárquico. J. Integrated Coastal Zone Manag. v. 12, n. 4, p. 509-520, 2012.

SÁ, C.A. et al. Obesidade, condição socioeconômica, hipertensão arterial no extremo oeste de Santa Catarina. Rev. Salud. Publica. v. 16, n. 2, p. 184-194, 2014.

SACKS, F.M. et al. DASH-SODIUM COLLABORATIVE RESEARCH GROUP. Effects on blood pressure of reduced dietary sodium and the Dietary Approaches to Stop Hypertension (DASH) diet. DASH-Sodium Collaborative Research Group. N. Engl. J. Med. v. 344, n. 1, p. 3-10, 2001.
SANTOS, P.V.C.J. et al. Perfil socioeconômico de pescadores do município da Raposa, Estado do Maranhão. Rev. Bras. Eng. Pesca. v. 6, n. 1, p. 1-19, 2011.

SBC- Sociedade Brasileira de Cardiologia. [Brazilian Society of Hypertension. Brazilian Society of Nephrology. VI Brazilian Guidelines on Hypertension. Arq.

Bras. Cardiol. v. 95, supl.1, p. 1-51, 2010.

SCHMIDT, M.I. et al. Chronic non-communicable diseases in Brazil: burden and current challenges. Lancet. v. 377, n. 9781, p. 1949-1961, 2011.

SIAB. Sistema De Informação da Atenção Básica 2011. Disponível em: <http://www2.datasus.gov.br/ SIAB/index.php> Acesso em: 23/06/2011

TALAEI, M. et al. Incident hypertension and its predictors: the Isfahan Cohort Study. J Hypertension. v. 32, n. 1, p. 30-38, 2014.

WHO. World Health Organization. Global Atlas on Cardiovascular Disease Prevention and Control. Mendis S, Puska P, Norrving B editors. Geneva: World Health Organization; 2011.

WHO. World Health Organization. Global Health Observatory (GHO) Data. Reised blood pressure Risk Factors, 2015. Disponível em: http://www.who. int/gho/ncd/risk_factors/blood_pressure_prevalence_text/en/. Acesso em 16 de Dezembro, 2015.

WHO. World Health Organization. Obesity: preventing and managing the global epidemic. Report WHO Consultation. Geneva; 2000. Technical Report Series, 894. 
2 Enfermeira pela Universidade Tiradentes - UNIT; Mestre em Saúde e Ambiente pela Universidade Tiradentes - UNIT, SE. E-mail: carlagrasiela. enfermeira@hotmail.com

3 Enfermeiro pela Universidade Tiradentes - UNIT, SE; Doutorando do Programa de Pós-graduação em Saúde e Ambiente, Universidade Tiradentes - UNIT, Aracaju, SE. E-mail: guigo-20@hotmail.com

Recebido em: 21 de Outubro de 2017 Avaliado em: 23 de Novembro de 2017 Aceito em: 2 de Dezembro de 2017
4 Enfermeira pela Universidade Tiradentes - UNIT, SE; Bolsista do Programa Institucional de Bolsas de Iniciação Científica da Universidade Tiradentes - PROBIC-UNIT. E-mail: regivalda_15@hotmail.com

5 Médico pela Universidade Federal de Sergipe - UFS; Docente do Programa de Pós-graduação em Saúde e Ambiente, Universidade Tiradentes - UNIT, SE. E-mail: franciscopradoreis@gmail.com 